\title{
Research on Timbre in Music and Its Application
}

\author{
Dawei $\mathrm{Yan}^{1,2, *}$ Bo $\mathrm{Tu}^{1,3}$ \\ ${ }^{1}$ School of Music, Central China Normal University, Wuhan, Hubei, China \\ ${ }^{2}$ The National Piano Society of China, Wuhan, Hubei, China \\ ${ }^{3}$ Hubei Musicians Association, Wuhan, Hubei, China \\ ${ }^{*}$ Corresponding author. Email: 568866639@qq.com
}

\begin{abstract}
Aiming at the original theory of the "timbre" problem, through sorting out the ins and outs of the concept of timbre, this article studies the relationship between timbre and music origin and the evolution process of the auditory system's perception of tone color, and gives new cognitive concepts of timbre in a narrow sense and a broad sense. It also analyzes the difference between timbre and sound, the relationship and difference between timbre and the other three elements of sound, as well as the relationship and difference between timbre in music and a series of musical elements derived from the other three elements of sound. The purpose is to achieve an overall grasp of the basic principles of cognitive timbre, further explore the value of the concept of timbre in music and its realization approaches, and provide theoretical support for the promotion of music teaching. At the same time, this article constructively enumerates some applications of the concept of timbre in piano performance, removes some obstacles in the process of timbre expression of music knowledge (music theory, harmony, musical form, etc.) and realizes the effective coupling of basic principles and practical perspectives.
\end{abstract}

Keywords: Objective quantity, Subjective quantity, Timbre in a narrow sense, Timbre in a broad sense.

\section{INTRODUCTION}

Timbre is not only a concept in music art, but also a concept in physical science, and there is an inseparable historical origin between them. If "music is the art of expressing emotion with sound", it is more accurate to say "music is the art of expressing emotion with timbre". What exactly is the timbre in music? An accurate and comprehensive understanding of timbre in music can not only clarify many vague understandings, but also clearly explain the relationship between art and science. This is also the reason and theme of this article. In the current era with the characteristics of expansion and speculation, the study of timbre can provide enlightenment for thinking about how to continuously reconstruct the unity of timbre theory and practice and the unity of scientific theory and historical logic, so as to further explore the value and application of the concept of

*Fund: 2021 Hubei Provincial Social Science Fund General Project "Research on the Inheritance and Development of Chinese Red Music Culture in the Past 100 Years Since the founding of the Party"(No. 2021047) timbre and provide theoretical guidance for promoting the construction of music teaching.

\section{THE ORIGIN OF THE CONCEPT OF TIMBRE}

The timbre of sound is a difficult concept to understand and recognize, and the issue of timbre needs to start from the source.

\subsection{Definition of Timbre}

In the history of Western music development, among the four elements of pitch, intensity, duration, and timbre, timbre is the latest element that people have discovered and defined. From the 16 th century to the 17 th century, some basic characteristics of sound were recognized by people, such as reflection, refraction, interference, diffraction and other phenomena of sound wave. In the music system, based on people's understanding of the three basic elements (frequency, sound intensity, and duration) that make up sound, some important laws of music, such as 12 equal 
temperament, polyphony, Harmony, and theory of music form, have been established one after another. The fact that the sound of the same pitch produced by different sounding bodies is not exactly the same has led to an in-depth study of the sensitivity of the human ear's frequency response. In 1843, for the first time the famous German physicist and electrician Georg Simon Ohm (also a music lover) used the mathematical Fourier transform to propose the theory that the human ear can decompose complex sounds into harmonic components and judge the sound quality (that is, timbre) according to the number and strength of the components".[1] Soon, another German scientist Hermann von Helmholtz used an acoustic resonator to test and analyze different sound spectra, confirming that the sound spectrum is the fundamental reason for timbre.[2] So the widely recognized last element of sound - "timbre" was confirmed. The original definition of timbre revealed that the generation of timbre is not only the vibration of objects on the physical level, but also the perception of people's auditory nerve system at the physiological and psychological levels. Subsequently, people have defined and interpreted timbre many times. In 1960, the American National Standards Institute made the definition of timbre more concrete as "Timbre is mainly determined by the stimulus of the sound spectrum to people, but it is also determined by the waveform, the relative intensity of the overtone, the frequency position of the spectrum, and the temporal stimulus of the spectrum to people". [3] This definition of timbre not only reiterates the physical vibration of timbre and the physiological human ear effect, but also further reveals the relationship between timbre, pitch, intensity, and duration. The definition of Encyclopedia of China Physics II is: "Timbre is a kind of auditory persistent phenomenon, combined by the objective quantity - the spectrum of the sound, and the subjective quantity - the human synaesthesia. This definition particularly emphasizes that timbre is a combination of objective quantity and subjective quantity.

\subsection{Timbre in Music}

Timbre in music is a theme that cannot be avoided in artistic performance and teaching practice. In order to clarify the role and application of timbre in music, the timbre is divided into:

\subsubsection{Timbre in a Narrow Sense}

Playing the same melody with piano and oboe makes it easy to distinguish between these two different musical instruments. Listening to a symphony, people can always hear the sounds of different musical instruments in the symphony orchestra. "He was born with a beautiful voice" is a typical application of the definition of timbre. Different musical instruments and people's throats produce their own unique spectrum. Timbre is an inherent attribute of the sounding body. Just like "you can't find two identical leaves in the world", the structure of any two people's throats cannot be $100 \%$ identical. Therefore, their sound spectrum is always different. The timbre is only used to distinguish sounding bodies. This relatively narrow, local application closely related to the definition of timbre is called the timbre in a narrow sense. People don't have to look at the tableau, but only rely on auditory judgment to change from abstract to concrete and produce a sense of tableau. But it must be emphasized that using the spectrum to distinguish between different sounding bodies does not mean that the timbre has no emotional color. For example, her voice is sweet, while her voice is a bit noisy; the voice of the trumpet is bright, the voice of the cello is rich and full, and so on. In music, it is precisely by taking advantage of the different emotional colors of the timbre of different sounding bodies that their applications are chosen.

\subsubsection{Timbre in a Broad Sense}

Theoretically, pure tone only has frequency, loudness and duration, but not the attribute of timbre. The timbre of a tone with certain frequency in the natural world is just a series of overtones superimposed on the pure tone image. This superimposed waveform envelope[4] is the timbre perceived by the human ear. Using several tones of different frequencies is like colour modulation in painting, and superimposing them together creates another timbre (such as chord, harmony). This rule was recognized by people before the concept of timbre was discovered, which was why it was later named timbre (color of tone) rather than something else when it was discovered.

Before the concept of timbre was discovered, some of the physical properties of light had already been mastered. That is, light is also an energy wave, and the visible spectrum wavelength (400760 nanometers) produces seven monochromatic lights of red, orange, yellow, green, blue, indigo, 
and violet from high to low. Any two kinds of coloured light can be combined into another coloured light by superimposing them together. Similarly, the audible sound spectrum (20-20000 $\mathrm{Hz}$ ) produces a series of pitches from low to high. Composers often use low-frequency sounds to depict deep and sad scenes, and high-frequency sounds to depict clear and fresh scenes. The ensemble of several sounds creates a new auditory experience.

Due to the similar physical properties of "sound" and "light", when people name the fundamental tone and overtone frequencies of a sound, they define them as the color of the tone timbre. Similarly, the application of timbre in the context of musical functions such as harmony, chord, mode, melody, and even musical form, which generate emotional colors, are derived from the promotion of note superimpositions, do not involve specific concepts and have a definite abstract meaning, is referred to as the timbre in a broad sense.

\subsubsection{The Auditory Effect of Timbre}

The definition of timbre emphasizes "the combination of synaesthesia", and timbre itself is inseparable from the perception of human ear. The stimulus received by the human ear receptors is transmitted to the cerebral cortex through nerve fibers, and corresponding emotions are generated through the effectors. In nature, the vibration of any sounding body can produce a series of harmonic tones. In the long process of human evolution, the auditory nerve system gradually adapts to the sound environment in nature. The auditory system has learned to receive, analyze, synthesize, and process various timbres of sounds within the frequency range that it can perceive, including various native harmonic timbres that belong to the category of music aesthetics, and produce corresponding emotions and scene perceptions in the cerebral cortex. The timbre of various sounds in the natural world and the human auditory system gradually establish various emotional and artistic connections. The earliest timbre that a baby can understand is the hypnotic humming of the mother. Before the production of language, people used shouts to convey information, created work songs in production, and expressed happiness with the sound of striking stone tools. The emotional effects of these timbres are not only for humans, but also for animals. Livestock will be very peaceful when they hear harmonious and pleasant music. When the eardrums of the livestock are stimulated by the high-frequency noise of the earthquake, it will cause a general turmoil. The effect of human physiological perception of timbre is the familiar sense of music. Although the races in the world (skin color, eye color, hair color, nose shape) are different, the genetic traits of perceiving timbre are common. This is the foundation of "music is the language of the world".

\section{THE OVERALL ANALYSIS OF THE BASIC PRINCIPLES OF TIMBRE}

Modern scientific methods, namely, the research methods of natural sciences, are based on "reductionism", using experimental, mathematical, and analytical methods, advocating "demonstration" and emphasizing "clarity". It is necessary to understand and recognize from multiple dimensions and levels. The study found that different versions of the definition of timbre, although the essence is the same, are very concise, and it is impossible to thoroughly analyze its connotation and extension, therefore, it needs to be understood and recognized from multiple dimensions and levels.

\subsection{Timbre Is a Combination of Subjective and Objective Quantities}

For a long time, some researchers have argued that timbre in music is an "aesthetic artistic concept other than physical properties", and separated the timbre in physics from the timbre in music for research.[5] The author believes that this research method is not scientific enough. If one can clarify the ins and outs of timbre, one can find that timbre is not only a concept in the category of art, but also a concept in the category of natural sciences. Timbre is the unity of opposites between the physical vibration of the sounding body and the human auditory perception, and it cannot be divided into two different events. Whether it is the timbre in a narrow sense or timbre in a broad sense, it is derived from physical vibration and human ear perception. For example, when people hear the cuckoo call, they perceive the cuckoo instead of other birds (timbre in a narrow sense), and at the same time perceive that "spring is coming" (timbre in a broad sense). In daily life, timbre can not only make listeners recognize the singer (timbre in a narrow sense), but also make people feel kind, sweet, affectionate and sincere (timbre in a broad sense). If there is a difference, it means that the 
focus is different. When a symphony plays birdsong, the performer shall choose the piccolo instead of the piano, focusing on the application of timbre in a narrow sense; when a performer uses only a piano to play a symphony (such as a symphony version of the piano recomposition), the audience shall pay attention to the generalized color of the piano's artistic conception, rather than the timbre of the sounding body in a narrow sense.

When a performer plays major triads and minor triads (physical level) on the piano, he can feel (music level) that: 1. The vibrating body is a piano; 2. The major triads can produce "dignified and brilliant mode color" and minor triads can produce "sorrowful and comforting mode color". While when a performer uses the violin to play the part of "transformation into buttefly" of "The Butterfly Lovers", the sounding body that people can perceive is the violin; the mode, beat, pitch, intensity, strength, phrase, etc. that constitute the melody are all objective quantities, and the poignant and earth-shaking emotions that people feel are subjective quantities. In other words, any timbre in music is a unity of opposites both on the physical and artistic levels.

\subsection{Timbre and Sound}

Judging from the known research materials, long before the concept of timbre was discovered, the timbre in music had existed objectively, and the "Harmony" that explained the law of timbre in music had also been established. The timbre at that time was replaced by "sound" or vocabulary describing timbre (such as: light, intense, soft, resplendent, etc.). So is the sound the timbre? What are the connections and differences between them?

In terms of structure and function, sound has four elements, namely pitch, intensity, duration and timbre. It can be seen from the spectrum and fluctuation image of the timbre that the timbre is composed of pitch, intensity, and duration. When people perceive the timbre, they indeed feel the pitch, intensity and duration. Recognizing the timbre of different sounding bodies depends on the frequency of the sounding body's fundamental tone plus characteristic overtones, and has nothing to do with the intensity and duration. No matter how great (how small) strength the performer uses (legato or staccato) to play (legato or staccato), people can recognize that the acoustical generator is a piano; the emotional timbre perceived by the human ear is closely related to the intensity and duration. For example, when portraying an oncoming train, the performer needs to increase his performance strength; when portraying a train going far gradually, the performer needs to use a decreasing strength; affectionate timbre often requires long-duration legato to express and so on.

If distinguished from the structure and nature: Firstly, the frequency (pitch) of sound is very wide. In addition to the sound wave frequency $(20 \mathrm{~Hz}-$ $20000 \mathrm{~Hz}$ ) that the human ear can perceive, it also includes ultrasonic and infrasonic wave frequencies. The frequency in the timbre is limited to the sound wave frequency that the human ear can perceive $(20 \mathrm{~Hz}-20000 \mathrm{~Hz})$. Secondly, the three elements of sound, namely, pitch, intensity, and duration, are objective, and do not rely on the perception of human ear as the premise of existence. While timbre, a sound attribute, must be based on human ear perception. The definition of timbre emphasizes subjectivity; pitch, intensity, and duration are the carriers of timbre; the perception of human ear is the necessary and sufficient condition for timbre. To be precise, timbre is not only an element of the sound, but also the part of the sound that the human ear can sensitively distinguish the spectrum. This is also the reason why sound can replace timbre in history. It's hoped that readers will remember that the perception of the human ear is a necessary and sufficient condition for the existence of timbre. Perception of what? Perception is the emotion/idea formed in the nerve center of the cerebral cortex. After the timbre is discovered and defined, both music creation and artistic performance have been further optimized, making the application of timbre more clear, precise, and more scientific. For example: when people hear a major triad played, which is better to answer with "This is the sound of a major triad" or "This is the timbre of a major triad"? Undoubtedly, the former is general, while the latter is clear and precise.

\subsection{Timbre and Musical Element}

Timbre is one of the elements that make up music. What is the relationship and difference between it and other elements that make up music (mode, rhythm, melody, phrase, harmony, etc.)?

Research has found that key and mode are derived musical elements of the sound pitch; note, rest, and phrase are derived musical elements of the sound duration; rhythm is a derived musical element of sound intensity and duration; melody is a derived musical element of sound pitch, intensity, duration, and so on. That is to say, all the elements that make up music except timbre are derived from 
the pitch, intensity, and duration of the sound. The timbre element in music coexists and dies with other elements, which is their interdependent relationship. Just as pitch, intensity, and duration are the carriers of timbre, other elements (mode, rhythm, melody, phrase, harmony, etc.) that make up music (except timbre) are carriers of timbre. The relationship between timbre and other elements of music are the relationship between the stowage and the carrier, which is their essential difference and should not be confused or replaced with each other.

Some people think that "Timbre has the function of deepening the artistic conception of music"[6] and "Timbre is the most important element of music", and some even propose "rewriting the definition of timbre" and so on. The over-exaggerated understanding and cognition of the concept of timbre are not supported by theory and practice. The "artistic conception of music" itself is the form of timbre. It is not logical to use timbre to deepen timbre. The deepening of the artistic conception of music can only be accomplished by the functional elements that carry timbre. Deepening a monophonic melody can only be done by means of configurating harmony, polyphony, etc., not the timbre itself. "The relationship between timbre and other musical elements is the relationship between the stowage and the carrier. There is no one more important than the other". Some exaggerated usages of the concept of timbre are related applications of timbre in a broad sense that have been mastered by people before the concept of timbre is discovered. That's all. For example: vertical harmony, chord and horizontal harmony progression are all objective quantities of musical elements; the former is the subjective quantity of instantaneous timbre like photos, and the latter is the subjective quantity of relatively complete continuous timbre (dynamic image). The relationship between timbre and other musical elements is the relationship between the stowage and the carrier; they are bundled together and cannot be separated; it is meaningless to talk about timbre without the carrier. The application of timbre cannot replace the other three elements of sound, nor can it replace various musical elements in music; discussing the issue of timbre should not only one-sidedly emphasize the artistic value while ignoring the theoretical support; no new definition of timbre has been found so far (including China and foreign countries) Some invalid researches and over-interpretations that one-sidedly exaggerate the function of timbre make the already difficult to understand timbre concept more complicated. It is necessary to understand the concept of timbre accurately and prudently, so as to provide a fundamental and original perspective for further thinking about "what timbre is". The core of this article is to remove the obstacles that perplex the application of timbre and related music theory knowledge, reshape the principle of integrity of the timbre concept, and practically apply the theoretical results to specific performance (and teaching) practice by means of clarifying the relationship between timbre and sound, and timbre and the constituent elements of music.

\section{THE APPLICATION OF TIMBRE IN PIANO PERFORMANCE}

In the analysis of the theory and performance background in piano works, the timbre problem needs to be sorted out in order to find the answer to the practical problem from the theoretical research.

\subsection{Grasping the Principal Contradiction and the Principal Aspect of a Contradiction}

In piano music, timbre and other elements are two aspects in a contradiction bundled together (reciprocal interdependence). In practice, the development and change of each event has the principal contradiction and the principal aspect of a contradiction that dominates and play a leading role. Grasping the principal contradiction and the principal aspect of a contradiction will promote the development of the event in the direction of the assumption.

When a child begins to learn the piano, music reading and flexible fingers practice are the principal contradictions in learning, and the timbre is the secondary contradiction. During the study of "Hanon", when learning the fingers practice, beat, strength, and note duration are the principal aspects of a contradiction. By grasping the principal aspects of a contradiction, the perfect timbre expected to be played will be obtained, and the flexibility of the five fingers will also be improved. When it comes to improvement based on the mastery of certain playing techniques, the timbre gradually rises to the principal aspect of a contradiction.

First, it's needed to improve performance techniques with musical emotions. When a performer is in a bad mood, it is difficult for him to play a bright and joyful etude; but when he practices an etude that expresses his depression, it will go smoothly. In other words, playing with the 
colors of the etude is the optimal way to learn and improve performance techniques. When learning new works, while clearly explaining all the functional elements in the music score, it is necessary to strive to accurately express the emotion and artistic conception of the music. After the performer fully understands and masters the timbre expression of the music, he can memorize and play ("memorize and play" is an advanced method to improve the sense of music and music memory). When the performer plays again with full emotion, a subconscious "divine key touch" will occur, which effectively improves playing techniques. Here, timbre is the principal aspect of a contradiction.

Second, when the performer listens to the performance and finds that the timbre is not right, he should find the reason from the musical elements that carry the timbre. When playing the homophony music, the sound intensity coordination between the main melody and the foil accompaniment parts runs through the music. Neither can the foil accompaniment parts annihilate the main part, nor can the foil accompaniment parts be played dispensable. Efforts should be made to pay attention to adjusting the playing strength of both hands at any time to obtain the best dynamic timbre effect and ensure that the main melody is perfectly presented in the multi-part of the homophony, so that the main melody is clear and full. It can be seen that the effective balance of sound intensity is the principal contradiction among many contradictions, and the sound intensity element that carries the main melody is the principal aspect of a contradiction. Only when the principal contradiction and the principal aspect of a contradiction are dealt with can the beautiful timbre be highlighted.

Third, "impromptu accompaniment" is one of the necessary skills for normal university students. The level of impromptu accompaniment ability reflects the degree to which students have mastered the comprehensive professional piano ability. The main task of impromptu accompaniment is to configure harmony for the singer. If the harmony is handled well, it can add lustre to the singing; it not, plus improper configuration time, improper intensity, etc., it will affect the singing effect. The timbre effect of harmony is the principal contradiction that plays a decisive role.

The accompanist must first be familiar with the overall artistic conception of the song, and then familiar with the primary chords, secondary dominants, and cadence instantaneous timbres that match the song, as well as the continuous timbres of broken chords and mode harmony. On this basis, with the use of harmony techniques, clever playing techniques, random configuration of intros, changeover portions, etc. for the song, one can perfectly achieve impromptu accompaniment. The beauty of the overall timbre should be implemented in a series of elements that describe the overall timbre, such as configuration time, strength, chord, broken chord, harmony, melody, etc. Especially, the configuration time and the control of strength are the principal aspects of a contradiction of the overall timbre effect. Only by handling these elements accurately and skillfully can impromptu accompaniment be effectively realized.

Fourth, during the performance, in addition to having a clear grasp of the scene, the image, the emotion and the style of the work, one should also discover and correct specific problems that affect the expression of timbre in a timely manner, such as wrong note and rhythm, inaccurate note duration, uneven strength, fingering error and so on. The performer needs to repeatedly play the corrected error until he can express the timbre perfectly. Experience has proved that even if the rhythm error of 0.1 second is corrected, unexpected timbre effects will be received. A performer must not start from the beginning easily when he encounters a problem. Focusing on the mistakes, accurate and repeated correction exercise is an effective way to get twice the result with half the effort.

\subsection{Using Theory to Guide Practice}

The use of "Harmony" is an effective means of breaking through the bottleneck of the interpretation of piano music timbre. "Harmony" is one of the greatest discoveries in the music history of mankind. Because of harmony, the colors of music can be so brilliant and rich. Here, the author would like to simply trace the history of "Harmony": From the ancient interval relationship to the use of the perfect consonance interval relationship to play a harmonious instantaneous timbre, the study of continuous timbre began. The musicians gave independence to the extended instantaneous timbre and produced a set of most basic counterpoint rules. Along with the demand for more colorful music, in 1722, French composer and theorist Rameau wrote the first version of "Harmony". This theory is still the basis of the study of tonal harmony. Afterwards, during The Romantic Period, the unessential note was 
introduced into "Harmony", which expanded the timbre nature of harmony. "At the end of the 19th century and the beginning of the 20th century, the impressionist composers represented by the French composer Debussy formed a set of impressionist harmony language". [7] Its harmony features paid more attention to color rather than function. Since modern times, because people's tolerance for diaphony has been further improved, the harmony theory of atonal music and the theory of jazz harmony have further broadened the color of harmony. Today, theories of harmony are still being developed and improved.

From above descriptions, it is not difficult to see that "Harmony" is a science that studies instantaneous timbre and deduces the law of continuous timbre according to its logical relationship, and its functionality all serves the color. The past, present and future of "Harmony" is to provide support for the creation of more beautiful musical color development. From the perspective of the timbre performance of harmony, harmony can also be regarded as "chromatics", but timbre cannot replace the function of "Harmony", so it cannot be renamed as "chromatics".

To play a piece of "Invention", students who have not yet learned harmony are often at a loss. But for students who have studied harmony, under the guidance of the teacher's demonstration, using the law of harmony, it will be easy for them to master the timbre expressed in the work. In teaching, when students cannot hear the emotions and artistic conceptions expressed by the music for a while, the simplest and most feasible way is to deal with them by means of harmony. First, the teacher should find out the mode in the processing unit and the various chords of the mode to help students carefully taste the instantaneous timbre presented by various chords as well as the continuous timbre presented by broken chords, mode scales and tonality,; then, combined with the composition background of the music, students need to weigh the various signs given by the composer in the music score, and carefully understand the meaning of timbre vocabulary. During the performance, students should taste the continuous timbre of the harmonic progression termination extension phrase by phrase and section by section. When the mode is switched, if it involves the collapse of the original tonal system and the establishment of a new tonal system, the harmony function will bring a sharp color contrast. After several repetitions, students can basically experience the general method of using "Harmony" to analyze the emotion and artistic conception expressed by music. After the "Harmony" is closely combined with the practice of piano learning, if things go on like this, through harmony analysis, the key points of concern of piano works such as style, period and technique can be solved together.

\subsection{Timbre and Performance Technique}

Piano performance technique is improved synchronously with the progress of piano manufacturing and the development of musical works. All performance methods, techniques and skills take the best timbre of the work as the criterion. Then, there is a one-to-one correspondence between the timbre expressed in the work and the performance technique.

"Quick playing technique" is often needed to shape artistic scenes such as crystal clear and smooth scenes and scenes of bees flying. In the fast operation of a single tone, the flexibility of knuckles and metacarpal joints is the key, crossing the thumb is the difficulty, and the height of the finger being bounced depends on the timbre environment. When playing at extreme speed, the performer's attention only needs to focus on the downward force of the fingertips, without raising the fingers (to reduce the time the fingertips spend running through the air). When running continuously upwards, the performer's right thumb should be quickly placed under his palm when he finishes touching the key to prepare for the next key touch. When going down, it's needed to be sure to synchronize the key touch of the performer's thumb with the translation of the palm in the downward direction, with the opposite motion in the left hand. The fast playing of chords and octaves mainly takes the wrist joint as the axis, swinging the entire palm to touch the key; it should be noted that while using the weight of the arm, the performer needs to make full use of the counterforce of the key on the fingertips to bounce the quickly relaxed palm; he also needs to adjust the height and speed of the key touch of the finger according to the color of the work. All of the above techniques require good relaxation of the limbs and good elasticity of the fingertips and key surfaces.

The presentation of soft, affectionate, and detailed phrase often requires "legato technique". The key points of the quality of the connection between sound and sound are: 1. Every time a key is touched, the performer must touch the key to the bottom, and the relaxed arm obtains the support of the key surface on the fingertips. 2. As the touching 
finger of the latter note brings the key to the bottom, the performer can feel the fingertips of the previous note being bounced by the key surface. The chord and octave legato technique depends on the needs of the timbre expressed. After playing the previous octave (or chord) with the wrist up, the performer can choose to keep one finger connected to the next octave (or chord) or to touch the next octave (or chord) connection quickly the moment the fingers all leave the key surface. The latter must borrow a pedal to complete the connection because it is impossible to avoid disconnecting the former and latter tones.

When depicting jumping, agitated, and lively colors, "staccato technique" is often used. Regarding the staccato of the wrist, it is important to emphasize here: While decisively touching the key to the bottom with the relaxed arm in concert with gravity, the performer must be sure to feel that the key surface bounces the relaxed wrist up through the fingertips. It's like a ball thrown to the ground is bounced off the ground.

In the art of piano performance, in addition to presenting the music inspirations, images, and emotions of the work to the audience with the color of the sound, the physical activities and facial expressions of the performers also simultaneously interpret the music inspirations, images, and emotions of the work. When playing mysterious, gentle, and fading content, the performer will sometimes lean over and listen to the colors played in the upward (or downward) direction. When depicting a magnificent and brilliant scene, the performer lifts his arms high with the help of the strong bounciness of the key surfaces. When the performer uses both hands to play violent and raging sections at the same time, from time to time, the audience will see the performer's buttocks being shaken away from the seat by the counterforce of key surfaces touched by the fingers and the feet on the ground. The expression of the performer is a natural expression of the interpretation of the work; even if his comprehension of the work is not yet in place, he should not take any actions that are irrelevant or contradictory to the content of the work.

\section{CONCLUSION}

In short, it is necessary to fully grasp the basic principles of scientific timbre, accurately define the role of timbre in music, deepen the understanding of the basic principles of timbre with a fundamental and original perspective and a stronger theoretical foundation, provide new enlightenments for solving new problems in the practice of timbre, and provide a certain theoretical reference for music education at the same time.

\section{AUTHORS' CONTRIBUTIONS}

Dawei Yan is responsible for experimental design, analysed data, and Bo $\mathrm{Tu}$ wrote the manuscript, contributed to revising and editing.

\section{REFERENCES}

[1] Ma Dayou, A Brief History of Acoustic Development $[\mathrm{J}]$. Cutting Edge Education, 2019 (05) 04

[2] Lin Jiaan, A Brief Talk on Physiological Optics [J]. Journal of Tianjin University of Technology, 1984 (S1) 19.

[3] Spectrum: a graph in which the fundamental tone and the amplitude of each overtone of the sound are arranged in order of frequency.

[4] Waveform envelope: "The so-called envelope detection is also called amplitude demodulation, and it has important applications in many fields.

[5] Wu Yuanhua. Interpretation of the research literature on music timbre since the reform and opening up $(1,2)[\mathrm{J}]$. Art Research, 2014 (02), 041

[6] Wang Dachang. On the timbre attributes and expressive functions of music [J]. Musicology in China, 1999 (04) 115.

[7] Yang Yue, On the Origin and Development of Harmony [J]. Literature, History and Art 2017 (07) 199. 